\title{
Selective Extirpation of Tattooed Lymph Node in Combination with Sentinel Lymph Node Biopsy in the Management of Node-Positive Breast Cancer Patients after Neoadjuvant Systemic Therapy
}

\author{
Lukas Dostalek Andrej Cerny Petra Saskova David Pavlista \\ Gynecologic Oncology Center, Department of Obstetrics and Gynecology, First Faculty of Medicine, \\ Charles University in Prague and General University Hospital in Prague, Prague, Czech Republic
}

\section{Keywords}

Breast cancer - Axilla - Carbon - Neoadjuvant chemotherapy . Sentinel lymph node

\begin{abstract}
Introduction: Axillary dissection has little diagnostic and therapeutic benefit in node-positive breast cancer patients in whom axillary disease has been completely eradicated after neoadjuvant chemotherapy (ypN0). We sought to assess the efficacy of an algorithm used for the identification of the ypNO patient consisting of intraoperative evaluation of sentinel and tattooed (initially positive) lymph nodes. Methods: Included were T1 and T2 breast cancer patients with 1-3 positive axillary lymph nodes marked with carbon who were referred for neoadjuvant chemotherapy followed by a surgery. Axillary dissection was performed only in the patients with residual axillary disease after neoadjuvant chemotherapy on ultrasound or with metastases described in the sentinel or tattooed lymph nodes either intraoperatively or in the final histology. Results: Out of 62 initially included node-positive patients, 15 (24\%) were spared axillary dissection. The detection rate of tattooed lymph nodes after neoadjuvant chemotherapy was $81 \%$. The ypN0 patients were identified with $91 \%$ sensitivity and $38 \%$ specificity using ultrasound and intraoperative assessment of both sentinel and tattooed lymph node according to the final histology. Discussion/Conclusion: Lymph node marking with carbon dye is a useful and cost-effective method, which can be successfully implemented in order to reduce
\end{abstract}

karger@karger.com www.karger.com/brc

Karger $\frac{1}{\%}$ BOPEN ACCESS
(C) 2021 The Author(s)

Published by S. Karger AG, Basel

This is an Open Access article licensed under the Creative Common Attribution-NonCommercial-4.0 International License (CC BY-NC) (http://www.karger.com/Services/OpenAccessLicense), applicable to the online version of the article only. Usage and distribution for commercial purposes requires written permission. the number of patients undergoing axillary dissection. Low specificity of the presented algorithm was caused mostly by the overestimation of residual axillary disease on ultrasound.

(C) 2021 The Author(s)

Published by S. Karger AG, Basel

\section{Introduction}

Traditionally, node-positive breast cancer patients have undergone neoadjuvant chemotherapy (NACT) followed by complete axillary staging - axillary dissection (AD), a procedure with considerable morbidities, such as chronic pain, decreased range of motion, and lymphedema [1]. Since a substantial proportion of the patients who present with a node-positive disease achieve complete eradication of the disease in the axilla after systemic treatment (ypN0), the question arises if it is still necessary to perform AD in all patients. Complete axillary staging reveals no further information in the ypN0 patients, and its therapeutic significance remains controversial. Yet, clinical identification of ypN0 patients appears to be an issue.

Unlike with clinically node-negative disease, AD cannot be simply replaced by sentinel lymph node biopsy (SLNB) in node-positive patients after NACT due to its high false-negative rate $[2,3]$. However, several methods have been described that may help reduce the false-negative rate to an acceptable level. One such method is marking the positive lymph node (LN) before administering 


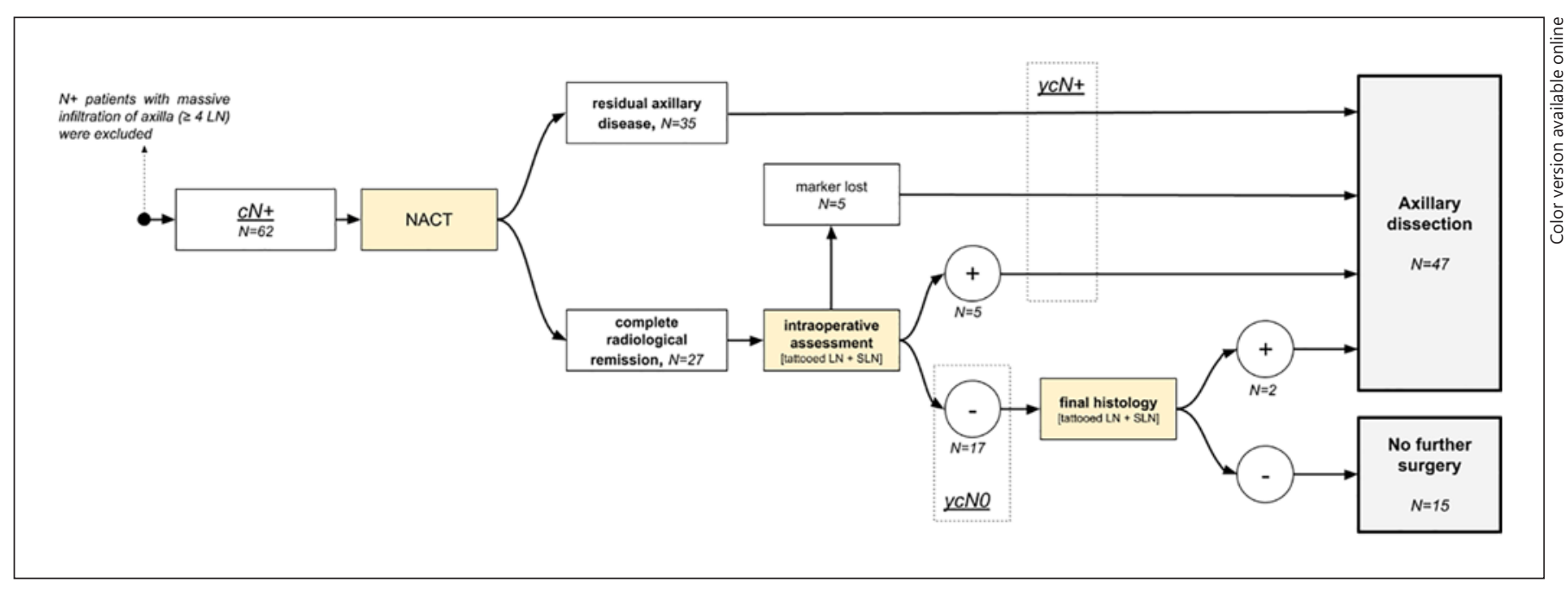

Fig. 1. Tattooed lymph node after NACT.

NACT and then performing selective extirpation in the course of the surgery.

Several markers have been used for this purpose, including carbon [4], a metallic clip [5], a seed with radioactive iodine [6] or a ferromagnetic seed (Magseed) [7]. Tattooing of axillary LN was proved to be a reliable and cost-effective method in recent trials [4, 8-10].

The primary aim of this study was to retrospectively analyze the efficacy of a novel management of node-positive breast cancer patients based on the identification of patients who achieve complete remission in the axilla after NACT (ypN0). These patients were identified using the combination of ultrasonographic restaging after NACT and intraoperative assessment of both sentinel LN and tattooed LN. Results of this restaging were compared with the final histology in terms of sensitivity and specificity. These, originally node-positive, patients, were spared $\mathrm{AD}$ in contrast to the commonly applied guidelines. We present experiences from the implementation of such management into everyday practice.

\section{Materials and Methods}

\section{Study Design and Patients}

We conducted a retrospective single-institution study that analyzed data from breast cancer patients treated between January 2018 and September 2020. The inclusion criteria were as follows: (i) bioptically verified invasive breast cancer, (ii) TNM stage T1 or T2, (iii) 1-3 suspicious axillary LN in initial radiological assessment (LN biopsy not required), (iv) administration of NACT followed by surgery. All patients were female. Patients with massive infiltration of the axilla ( $\geq 4 \mathrm{LN}$ ) and those with distant metastases were excluded.

Local clinical staging consisted of a physical examination, ultrasound, and mammography in combination with breast MRI in selected cases.
Positive LN were marked using carbon in all patients, and then NACT was administered. A radiological reassessment, based on the axillary ultrasound, was performed after completion of NACT. Patients with residual axillary disease were referred for $\mathrm{AD}$, and the rest was referred for extirpation of marked LN and SLNB.

\section{Marking}

LN marking occurred before the start of NACT. The selection of specific LN was based on sonographic characteristics: a finding of generalized or focal thickening ( $>3 \mathrm{~mm}[11])$ of the nodal cortex, a disparity in the size compared with other LN, a rounded appearance, or effacement of the node fatty hilum. Marking was performed with an injection of $0.1-0.5 \mathrm{~mL}$ of $4 \%$ solution of Carbo activatus (Carbosorb, IMUNA PHARM, Sarisske Michalany, Slovakia) into the cortex of the selected $\mathrm{LN}$ and the adjacent soft tissue. Each suspicious LN was marked separately. Additionally, a deposit of carbon was left in the tissue around the LN to mark the packet in the cases of multiple involved LN localized close to each other.

\section{Surgery}

Surgeries were performed by 1 of 3 experienced breast surgeons. Sentinel LN procedures consisted of preoperative periareolar injection of radioactive colloid ${ }^{99 \mathrm{~m}} \mathrm{Tc}$ - SentiScint, MediRadiopharma Ltd., Hungary, $4 \times 20 \mathrm{MBq}$ ) the day before the surgery. Sentinel LN and marked LN were identified after the opening of the axilla and submitted for frozen section assessment (see Fig. 1). In cases of intraoperatively reported LN infiltration or unsuccessful identification of marked LN, AD was completed. Otherwise, no further surgery was indicated. AD consisted of removal of all available LN from the first and second levels of the axilla.

\section{Histopathology}

Fresh tissue labeled "sentinel LN and marked LN" was delivered without fixation for intraoperative evaluation. The specimens were examined to determine the size and number of individual nodes. Grossly metastatic nodes were sectioned. The LN that appeared normal were cut perpendicularly to their long axis, and one half of each node was then examined in a frozen section on one level after staining with hematoxylin and eosin (HE). The other half of the node was immediately fixed. Finally, both LN halves were sectioned at $2-\mathrm{mm}$ intervals, and the entire LN was submitted 
Table 1. Characteristics of the whole cohort and subgroups of patients clustered according to the result of the assessment after NACT consisting of ultrasonographic restaging and intraoperative evaluation of sentinel and tattooed $\mathrm{LN}$ (ycN)

\begin{tabular}{|c|c|c|c|c|}
\hline & Whole cohort & ycN0 & $\mathrm{ycN}+$ & $p$ value \\
\hline Number & 62 & $17(27)$ & $45(73)$ & \\
\hline Age, years & $52.9 \pm 15.2$ & $52.2 \pm 15.6$ & $55.2 \pm 14.1$ & 0.70 \\
\hline \multicolumn{5}{|l|}{ Histotype } \\
\hline NST & 55 (89) & $16(94)$ & 39 (87) & 0.41 \\
\hline Lobular & $7(12)$ & $1(6)$ & $6(14)$ & \\
\hline \multicolumn{5}{|l|}{ Stage (from the final histology) } \\
\hline урТ0 & $14(23)$ & $5(29)$ & $9(20)$ & 0.23 \\
\hline ypTis & $3(5)$ & $2(12)$ & $1(2)$ & \\
\hline ypT1 & $25(40)$ & $7(41)$ & $18(40)$ & \\
\hline урТ2 & $20(32)$ & $3(18)$ & $17(38)$ & \\
\hline Mean initial tumor size, $\mathrm{mm}$ & $18.5 \pm 13.5$ & $14.6 \pm 13.3$ & $19.9 \pm 13.4$ & 0.37 \\
\hline \multicolumn{5}{|l|}{ Grade } \\
\hline 1 & $5(8)$ & $1(6)$ & $4(9)$ & 0.19 \\
\hline 2 & $25(40)$ & $10(59)$ & $15(33)$ & \\
\hline 3 & $32(52)$ & $6(35)$ & $26(58)$ & \\
\hline $\mathrm{ER}+$ & $25(40)$ & $7(41)$ & $18(40)$ & 0.93 \\
\hline $\mathrm{PR}+$ & $15(24)$ & $5(29)$ & $10(22)$ & 0.56 \\
\hline HER2/neu+ & $12(19)$ & $2(12)$ & $10(22)$ & 0.35 \\
\hline $\mathrm{Ki}-67, \%$ & $42.1 \pm 29.8$ & $43.2 \pm 28.2$ & $38.9 \pm 34.8$ & 0.79 \\
\hline Number of patients undergoing BCS & $33(53)$ & $11(65)$ & $22(49)$ & 0.27 \\
\hline Number of patients with positive LN in the final histology & $23(37)$ & $2(12)$ & $21(47)$ & 0.01 \\
\hline Number of patients with negative LN in the final histology & $39(63)$ & $15(88)$ & $24(53)$ & \\
\hline Spared from $\mathrm{AD}, \%$ & & 88 & 0 & \\
\hline Mean length of surgery & $68.9 \pm 21.1$ & $57.4 \pm 12.7$ & $73.2 \pm 22.1$ & 0.03 \\
\hline Mean number of acquired LN & $10.1 \pm 6.4$ & $4.1 \pm 3.3$ & $12.8 \pm 5.4$ & $<0.01$ \\
\hline
\end{tabular}

Numbers are indicated with percentages in parentheses. $y c N 0$, patients with negative ultrasound after neoadjuvant chemotherapy (NACT) and negative intraoperative assessment of both tattooed and sentinel lymph nodes (LN); ycN+, patients with residual axillary disease on ultrasound after NACT, patients with positive intraoperative assessment of tattooed or sentinel LN or patients in whom tattooed LN could not be intraoperatively identified; $p$ values assessed by $\chi^{2}$ and Kruskal-Wallis test; NST, no special type; ER+, estrogen receptor positivity; $\mathrm{PR}+$, progesteron receptor positivity; BCS, breast-conserving surgery.

for routine processing and HE staining. Any LN found negative upon routine examination was further examined by an ultrastaging protocol.

This protocol consisted of 2 consecutive sections ( $4 \mu \mathrm{m}$ thick) obtained in regular $150-\mu \mathrm{m}$ intervals, which were cut from each paraffin block in 4 levels. The first section was stained with HE, and the second section was examined immunohistochemically with an antibody to cytokeratins (AE1/AE3; 1:50 dilution, Dako, Glostrup, Denmark). An immunohistochemical examination was performed using the avidin-biotin complex method (Ventana ES autostainer, Ventana, Medical Systems, Tucson, AZ, USA).

\section{Further Clinical Management}

Patients were divided into 2 subgroups for the purposes of this analysis. Patients with no residual axillary disease after NACT assessed by ultrasound and intraoperative assessment of tattooed and sentinel LN were labeled as ycN0. The remaining patients (with residual disease on ultrasound or those with intraoperatively reported LN positivity) were clustered into the $\mathrm{ycN}+$ subgroup. Patients in whom the tattooed LN was not identified were assigned to the $\mathrm{ycN}+$ group.

Interval $\mathrm{AD}$ was completed in the patients who underwent marked LN extirpation and SLNB with negative intraoperative frozen section evaluation and nodal metastases described the final histology.

Tattooed Lymph Node and Sentinel

Lymph Node Biopsy in the N+Patient

\section{Data Analyses}

Standard measures of summary statistics were used to describe primary data: relative and absolute frequencies and arithmetic mean supplied with the SD of the mean.

The efficacy of the presented novel management of the nodepositive breast cancer patients was evaluated by calculating the sensitivity, specificity, positive predictive value, negative predictive value, positive likelihood ratio, and negative likelihood ratio. The result of ultrasonographic reassessment with or without intraoperative assessment of tattooed and sentinel LN was used as a unit of analysis, and the final histology result as a reference standard method (see Table 2).

The $\chi^{2}$ test was used to compare the groups in the parametric categories, and the Kruskal-Wallis test was used to compare the groups in the categories where the continual variables were provided. A value of $p=0.05$ was used as the limit of statistical significance in all the analyses performed.

\section{Results}

In all, 62 patients were retrospectively evaluated. Patients with invasive carcinoma of no special type comprised $89 \%$ of the study population. The rest of the cohort 


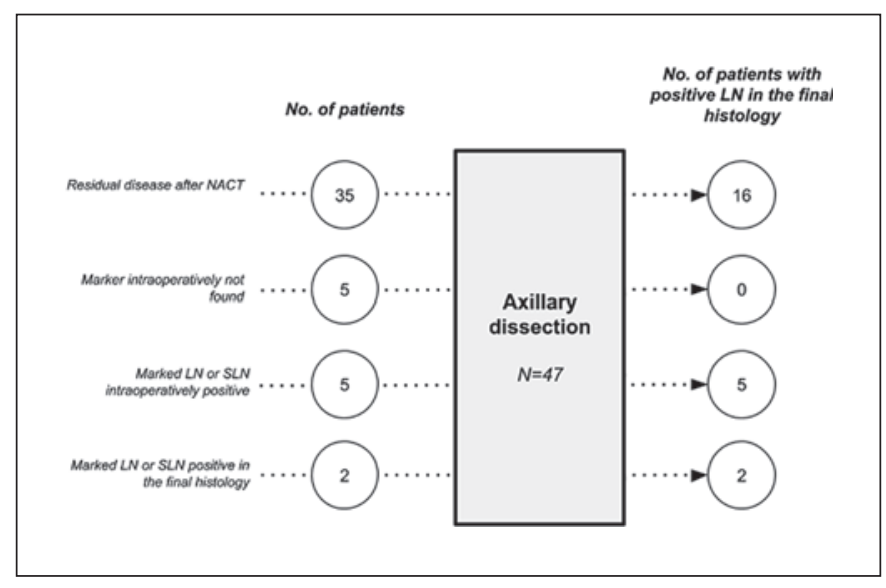

Fig. 2. Treatment scheme of $\mathrm{N}+$ breast cancer patients.

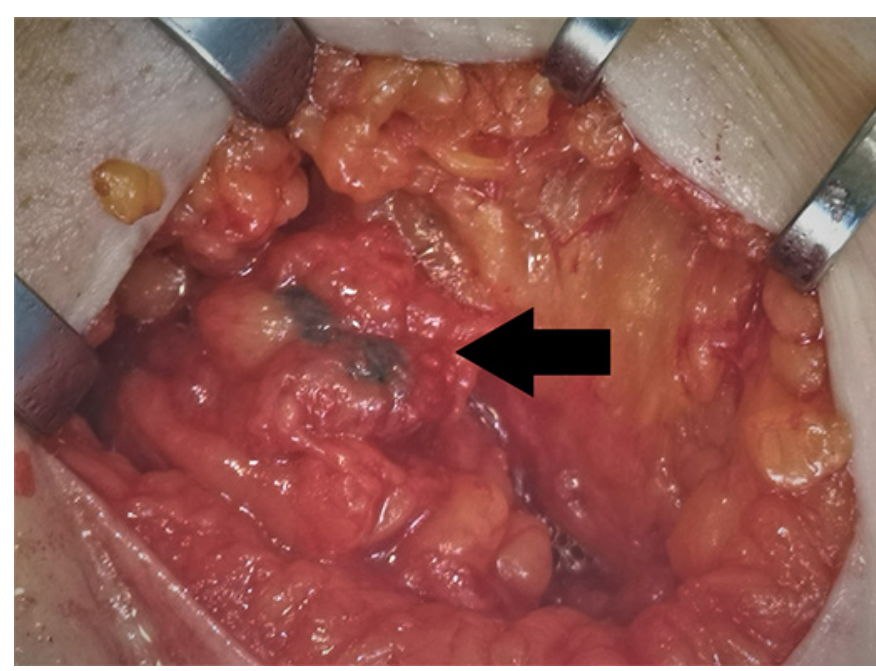

Fig. 3. Nodal status after axillary dissection - final histology.

had invasive lobular carcinoma (12\%; Table 1). All patients had infiltration of axillary LN and were referred for NACT.

Ultrasonographic reassessment after the administration of NACT showed no residual axillary disease (complete response) in 27 patients (44\%); these were indicated for extirpation of tattooed LN and SLNB (Fig. 2). The remaining 35 patients with residual nodal infiltration (who had axillary partial response, stable disease, or progression) were referred for $\mathrm{AD}$.

The surgeon was unable to localize the carbon-marked LN in 5 patients (out of 27 - detection rate $81 \%$ ). Nodal metastases were intraoperatively reported in 5 patients; additionally, in 2 patients $\mathrm{LN}$ infiltration was described in the final histology. AD was completed in all these $12 \mathrm{pa}$ tients. Out of 22 patients with successful intraoperative identification of marked LN, tattooed and sentinel LN were matching in 12 patients (55\%). In total, $\mathrm{AD}$ was performed in 47 patients (76\%), while 15 patients (24\%) un-
Table 2. Efficacy of ultrasonographic restaging after neoadjuvant chemotherapy with or without the intraoperative assessment of sentinel and tattooed lymph nodes (LN)

\begin{tabular}{lcc}
\hline & $\begin{array}{l}\text { Ultrasound } \\
\text { only, \% }\end{array}$ & $\begin{array}{l}\text { Ultrasound } \\
\text { with LN, \% }\end{array}$ \\
\hline Sensitivity & 70 & 91 \\
Specificity & 51 & 38 \\
Positive predictive value & 46 & 47 \\
Negative predictive value & 74 & 88 \\
Positive likehood ratio & 1.43 & 1.48 \\
Negative likehood ratio & 0.59 & 0.23 \\
\hline
\end{tabular}

The final histology result is used as a reference standard method.

derwent extirpation of tattooed LN and SLNB only (Fig. 2)

Of the 47 patients who underwent AD, 23 (49\%) had at least one positive LN in the final histology (Fig. 3). Among 35 patients who were directly referred for AD due to residual axillary disease after NACT, 16 (46\%) had nodal metastases in the final histology (Fig. 3). The sensitivity and specificity of axillary ultrasonographic restaging according to the result of the final histology was 70 and $51 \%$, respectively. The sensitivity rose to $91 \%$ with addition of intraoperative assessment of both sentinel and tattooed LN. However, specificity dropped to $38 \%$ due to the patients in whom the carbon tracer was not identified during the operation (Table 2). None of these 5 patients had positive axillary LN in the final histology.

The mean number of $\mathrm{LN}$ removed in the patients from the $y c N 0$ group (including data from the interval $\mathrm{AD}$ performed in the patients with positive LN identified in the final histology) was significantly lower than in the rest of the cohort $(\mathrm{ycN}+; 4.1$ vs. $12.8, p<0.01)$. The surgeries were shorter in the ycN0 group (extirpation of sentinel and tattooed LN) than in the ycN+ group (57.4 vs. 73.2 $\min , p=0.03$ ). We observed no difference in the distribution of histological types or tumor stages between the $\mathrm{ycN} 0$ and $\mathrm{ycN}+$ groups (Table 1).

Patients in the ycN0 group (those with negative ultrasonographic restaging after NACT and negative intraoperative assessment of both tattooed and sentinel LN) had significantly fewer positive LN in the final histology than the rest of the group $(\mathrm{ycN}+) .88 \%$ of $\mathrm{ycN} 0$ patients were spared AD.

\section{Discussion/Conclusion}

In this retrospective study of 62 node-positive breast cancer patients who were referred for NACT followed by surgery, 15 patients (20\%) were spared AD thanks to se- 
lective extirpation of the tattooed LN. The sensitivity of axillary restaging after NACT considerably improved with the implementation of intraoperative assessment of tattooed and sentinel LN (from 70 to 91\%). However, we also observed a decrease in specificity (51 and 38\%, respectively) due to an overestimation of residual axillary disease on ultrasound and suboptimal detection rate of tattooed LN (81\%). Both these factors contributed to the number of patients who underwent $\mathrm{AD}$ and had no positive LN described in the final histology.

According to the current NCCN guidelines, preoperative systemic treatment is preferred in patients with nodepositive disease likely to become node-negative after NACT [12]. As a complete axillary remission after NACT has been confirmed as a prognostic marker in breast cancer patients $[13,14]$, the treatment strategy should aim for the complete eradication of the disease in the axilla with NACT to improve the prognosis. NACT has been shown to downstage nodal status significantly in at least $20 \%$ of patients $[3,15,16]$. This number is increasing with improvements in the targeted systemic therapies.

Until recently, standard practice was to perform $\mathrm{AD}$ in all node-positive patients, even in those who achieved complete radiological remission in the axilla after NACT (ycN0). Despite the fact that AD is associated with considerable morbidities such as chronic pain, decreased range of motion, and lymphedema [1], identifying patients with no residual disease in the axilla who may not require $\mathrm{AD}$ has been a challenge.

The feasibility of SLNB after NACT remains controversial. The main concern is that tumor eradication after NACT could alter lymphatic drainage and lead to an increased false-negative rate of SLNB. In the context of SLNB, the false-negative rate (the proportion of patients with negative sentinel LN among patients with positive LN) is an important measure of procedural accuracy. Several recent trials have evaluated this question in a prospective fashion.

Boughey et al. [2] set a false-negative rate of $10 \%$ as an accepted cutoff rate, in the ACOSOG Z1071 trial investigating the implementation of SLNB after NACT. This was based on trials dealing with clinically node-negative disease. Out of $525 \mathrm{cN} 1$ prospectively analyzed patients after NACT who underwent both AD and SLNB and in whom 2 or more sentinel LN were identified, there were 310 patients with residual nodal disease. Of these, disease was confined only to the nodes removed on $\mathrm{AD}$ in $39 \mathrm{pa}-$ tients. A false-negative rate of $12.6 \%$ exceeded the predefined limit [2]. Similarly, there were 592 initially nodepositive patients who received NACT, followed by both SLNB and AD analyzed in the German-Austrian trial SENTINA [3]. All these patients achieved complete axillary remission and were downstaged to ycN0 after the NACT (arm C). The false-negative rate of SLNB reached $14.2 \%$ in this cohort.

Tattooed Lymph Node and Sentinel

Lymph Node Biopsy in the N+Patient
There are several methods that can contribute to the reduction of the false-negative rate, such as the implementation of immunohistochemistry [17], the use of dual tracers, the retrieval of a higher number of sentinel LN [2], or marking the positive LN before administration of NACT. The last modality was evaluated in a subgroup of 170 patients from the ACOSOG Z1071 trial, in whom a metal clip was placed in the $\mathrm{LN}$ at the time of biopsy. The false-negative rate dropped to $6.8 \%$ among the 107 patients in whom the clip was found in the sentinel LN [5].

A radioactive iodine $\left({ }^{125} \mathrm{I}\right)$ marker was used to mark positive LN at the time of diagnostic biopsy in a Dutch trial [6]. It was then left in situ until the breast surgery, which included its selective extirpation (MARI procedure). The marked node was identified successfully in $97 \%$ of patients, with a false-negative rate of $7 \%$. It is important to stress that the authors did not implement SLNB, which remains controversial. In a similar American trial, 85 patients underwent targeted axillary dissection (TAD-SLNB as well as extirpation of the $\mathrm{LN}$ with ${ }^{125} \mathrm{I}$ marker) followed by conventional $\mathrm{AD}$. The false-negative rate for both sentinel $\mathrm{LN}$ and marked LN was only $2 \%$ [18]. In the same paper, the retrospective analysis of 112 patients with clip placed in the positive LN before the administration of NACT who underwent SLNB followed by $\mathrm{AD}$ showed only 1 false-negative patient (with metastasis in other LN than the sentinel or clipped one) and 44 patients with no residual disease in the axilla (ypN0). The negative predictive value was $98 \%$. LN marking with carbon was evaluated in a trial of 12 patients who underwent NACT followed by a surgery. LN were marked before the administration of NACT, and the tattooed LN was intraoperatively successfully identified in all 12 patients [19]. These results are comparable with the $97 \%$ detection rate of the ${ }^{125}$ I seeds used in the MARI procedure [6]. The tattooed LN was intraoperatively identified in $82 \%$ of patients in a recently published multicenter trial. The detection rate was significantly lower in the patients after NACT compared with those undergoing primary surgery (86 vs. $64 \%, p=0.03$ ) [4].

Algorithm used for the identification of ypN0 patients was based on the assessment of both sentinel and tattooed $\mathrm{LN}$ in the current trial. Furthermore, all patients were triaged using ultrasound after NACT, and those with residual disease were referred directly for $\mathrm{AD}$. We observed high sensitivity (91\%) of such management arising from the fact that only 2 patients with negative axillary ultrasound after NACT and negative intraoperative assessment of tattooed and sentinel LN had positive LN in the final histology. On the other hand, a high proportion of false-positive cases described on the ultrasound after NACT (together with patients in whom the carbon tracer was intraoperatively not identified) caused low specificity (38\%) and a high number of patients who underwent $\mathrm{AD}$ 
(76\% of the whole cohort). The main limitation of these numbers lies in the fact that $\mathrm{AD}$ was not performed in the whole cohort. Node-negative patients (true negative subgroup) underwent SLNB and extirpation of marked LN only. However, given the high negative predictive value (98\%) of a similar algorithm described by Caudle et al. [18] using assessment of both marked and sentinel LN, we consider patients with negative intraoperative assessment of sentinel and tattooed LN (confirmed by the final histology) to be node-negative.

The highest chance of finding the initially positive LN even in case of inaccurate placement of the carbon tracer is in the situation when the marked and sentinel LN are identical. The only factor described to be associated with this concordance is the presence of 3 and less abnormal $\mathrm{LN}$ on the initial ultrasound [18]. From this reason we adopted this criterion in our algorithm.

The main limitation of our study is the fact that the nodal status $(\mathrm{cN})$ was initially not bioptically verified. This may mean that some of the patients labeled as false positive in the ultrasonographic restaging after NACT were not node positive at all. However, missing the LN metastasis during the biopsy would label such a patient as node negative, with potentially adverse consequences. Biopsy from a positive $\mathrm{LN}$ was not routinely performed in the presented trial. Another limitation is the retrospective and unicentric design of the presented trial. The study protocol was designed to evaluate the pilot phase of this novel approach implemented in a single center. Therefore, the presented results need to be validated in a multicentric study performed in the larger cohort, independently on the site-specific issues of radiodiagnostics and surgery. Only a prospective trial assessing the data on oncological safety (recurrence-free survival, etc.) can justify the permanent change in the management of the nodepositive breast cancer patients with complete eradication of the axillary disease after NACT.

Tattooing of the positive LN and its selective extirpation and intraoperative assessment in combination with
SLNB is a simple and cost-effective method which increases the sensitivity of axillary restaging after NACT. The presented algorithm can be effectively used to identify the patients with complete remission in the axilla who do not profit from $\mathrm{AD}$. In the current study, $24 \%$ of initially node-positive patients were spared AD. On the other hand, we observed only $38 \%$ specificity. This can be attributed to the overestimation of axillary disease both before and after the administration of NACT.

\section{Acknowledgments}

This work was supported by a grant from Charles University in Prague (UNCE 204065 and PROGRES Q28/LF1).

\section{Statement of Ethics}

Due to the retrospective nature of the study, the need for informed consent was waived by the Institutional Review board. Ethical approval for this study (No. 734/20 C-IV) was provided by the Ethics Committee of the General University Hospital, Prague.

\section{Conflict of Interest Statement}

The authors have no conflicts of interest to declare.

\section{Funding Sources}

The authors did not receive any funding for this study.

\section{Author Contributions}

L.D.: data curation; formal analysis; methodology; project administration; writing - original draft; investigation. A.C.: investigation. P.S.: investigation. D.P.: conceptualization; formal analysis; methodology; project administration; supervision; investigation.

\section{References}

1 Donker M, van Tienhoven G, Straver ME, Meijnen P, van de Velde CJ, Mansel RE, et al. Radiotherapy or surgery of the axilla after a positive sentinel node in breast cancer (EORTC 10981-22023 AMAROS): a randomised, multicentre, open-label, phase 3 non-inferiority trial. Lancet Oncol. 2014 Nov; 15(12):1303-10.

2 Boughey JC, Suman VJ, Mittendorf EA, Ahrendt GM, Wilke LG, Taback B, et al.; Alliance for Clinical Trials in Oncology. Sentinel lymph node surgery after neoadjuvant chemotherapy in patients with node-positive breast cancer: the ACOSOG Z1071 (Alliance) clinical trial. JAMA. 2013 Oct;310(14):1455-61.
3 Kuehn T, Bauerfeind I, Fehm T, Fleige B, Hausschild M, Helms G, et al. Sentinellymph-node biopsy in patients with breast cancer before and after neoadjuvant chemotherapy (SENTINA): a prospective, multicentre cohort study. Lancet Oncol. 2013 Jun; 14(7):609-18.

4 Goyal A, Puri S, Marshall A, Valassiadou K, Hoosein MM, Carmichael AR, et al. A multicentre prospective feasibility study of carbon dye tattooing of biopsied axillary node and surgical localisation in breast cancer patients. Breast Cancer Res Treat. 2020, Online ahead of print.
5 Boughey JC, Ballman KV, Le-Petross HT, McCall LM, Mittendorf EA, Ahrendt GM, et al. Identification and Resection of Clipped Node Decreases the False-negative Rate of Sentinel Lymph Node Surgery in Patients Presenting With Node-positive Breast Cancer (T0-T4, N1-N2) Who Receive Neoadjuvant Chemotherapy: Results From ACOSOG Z1071 (Alliance). Ann Surg. 2016 Apr;263(4):802-7.

6 Straver ME, Loo CE, Alderliesten T, Rutgers EJ, Vrancken Peeters MT. Marking the axilla with radioactive iodine seeds (MARI procedure) may reduce the need for axillary dissection after neoadjuvant chemotherapy for breast cancer. Br J Surg. 2010 Aug;97(8):1226-31. 
7 Greenwood HI, Wong JM, Mukhtar RA, Alvarado MD, Price ER. Feasibility of Magnetic Seeds for Preoperative Localization of Axillary Lymph Nodes in Breast Cancer Treatment. AJR Am J Roentgenol. 2019 Oct;213(4): 953-7.

8 Patel R, MacKerricher W, Tsai J, Choy N, Lipson J, Ikeda D, et al. Pretreatment Tattoo Marking of Suspicious Axillary Lymph Nodes: Reliability and Correlation with Sentinel Lymph Node. Ann Surg Oncol. 2019 Aug;26(8):2452-8.

9 Park S, Koo JS, Kim GM, Sohn J, Kim SI, Cho YU, et al. Feasibility of Charcoal Tattooing of Cytology-Proven Metastatic Axillary Lymph Node at Diagnosis and Sentinel Lymph Node Biopsy after Neoadjuvant Chemotherapy in Breast Cancer Patients. Cancer Res Treat. 2018 Jul;50(3):801-12.

10 Natsiopoulos I, Intzes S, Liappis T, Zarampoukas K, Zarampoukas T, Zacharopoulou V, et al. Axillary lymph node tattooing and targeted axillary dissection in breast cancer patients who presented as $\mathrm{cN}+$ before neoadjuvant chemotherapy and became cN0 after treatment. Clin Breast Cancer. 2019 Jun; 19(3):208-15.
11 Stachs A, Thi AT, Dieterich M, Stubert J, Hartmann S, Glass Ä, et al. Assessment of Ultrasound Features Predicting Axillary Nodal Metastasis in Breast Cancer: The Impact of Cortical Thickness. Ultrasound Int Open. 2015 Jul;1(1):E19-24.

12 Gradishar WJ, Anderson BO, Abraham J, Aft $\mathrm{R}$, Agnese D, Allison $\mathrm{KH}$, et al. Breast Cancer, Version 3.2020, NCCN Clinical Practice Guidelines in Oncology. J Natl Compr Canc Netw. 2020 Apr;18(4):452-78.

13 von Minckwitz G, Untch M, Blohmer JU, Costa SD, Eidtmann H, Fasching PA, et al. Definition and impact of pathologic complete response on prognosis after neoadjuvant chemotherapy in various intrinsic breast cancer subtypes. J Clin Oncol. 2012 May;30(15): 1796-804.

14 Hayashi N, Takahashi Y, Matsuda N, Tsunoda H, Yoshida A, Suzuki K, et al. The Prognostic Effect of Changes in Tumor Stage and Nodal Status After Neoadjuvant Chemotherapy in Each Primary Breast Cancer Subtype. Clin Breast Cancer. 2018 Apr;18(2):e219-29.

15 Fisher B, Brown A, Mamounas E, Wieand S, Robidoux A, Margolese RG, et al. Effect of preoperative chemotherapy on local-regional disease in women with operable breast cancer: findings from National Surgical Adjuvant Breast and Bowel Project B-18. J Clin Oncol. 1997 Jul;15(7):2483-93.
16 Kuerer HM, Sahin AA, Hunt KK, Newman LA, Breslin TM, Ames FC, et al. Incidence and impact of documented eradication of breast cancer axillary lymph node metastases before surgery in patients treated with neoadjuvant chemotherapy. Ann Surg. 1999 Jul;230(1): 72-8.

17 Fu JF, Chen HL, Yang J, Yi CH, Zheng S. Feasibility and accuracy of sentinel lymph node biopsy in clinically node-positive breast cancer after neoadjuvant chemotherapy: a metaanalysis. PLoS One. 2014 Sep;9(9):e105316.

18 Caudle AS, Yang WT, Krishnamurthy S, Mittendorf EA, Black DM, Gilcrease MZ, et al. Improved axillary evaluation following neoadjuvant therapy for patients with node-positive breast cancer using selective evaluation of clipped nodes: implementation of targeted axillary dissection. J Clin Oncol. 2016 Apr; 34(10):1072-8.

19 Choy N, Lipson J, Porter C, Ozawa M, Kieryn $\mathrm{A}, \mathrm{Pal} \mathrm{S}$, et al. Initial results with preoperative tattooing of biopsied axillary lymph nodes and correlation to sentinel lymph nodes in breast cancer patients. Ann Surg Oncol. 2015 Feb;22(2):377-82. 